\title{
AUTONOMOUS EXCAVATION USING HYDRAULIC MOBILE MACHINE RETROFITTED WITH ELECTRONIC CONTROL SYSTEM
}

\author{
Otso KARHU, Jarno UUSISALO, Janne HONKAKORPI and Kalevi HUHTALA \\ Department of Intelligent Hydraulics and Automation \\ Tampere University of Technology \\ P.O.Box 589, 33101 Tampere, Finland \\ (E-mail: otso.karhu@tut.fi)
}

\begin{abstract}
Autonomous functions are a common research topic in robotics. However, the robots typically have limited operating conditions and they can only handle relatively small loads. Many autonomous control concepts are applicable to actual construction machinery. The benefits would be considerable regarding efficiency, safety, and operator comfort. The research projects of this field have often led to systems with cost and complexity too high for most applications. This paper presents an implementation of a cost-effective distributed control system that is retrofitted to a small hydraulic excavator. The system can be used to perform simple operator assisting functions but also to develop autonomous excavation.

The hardware of the system consists of cost-effective joint angle sensors and a flexible microcontroller unit. Control schemes that produce autonomous functions are discussed and tested with the excavator. These include position controllers for conventional mobile proportional hydraulics. The aim is to find computationally simple but flexible algorithms for autonomous excavation. The results of the preliminary tests are presented. Future improvements are discussed.
\end{abstract}

\section{KEY WORDS}

Autonomous machines, Control, Excavator, Mobile hydraulics

\section{NOMENCLATURE}

CAN : Controller Area Network

MEMS: $\quad$ Micro Electrical Mechanical System

PWM : Pulse Width Modulation

$\theta_{1} \quad:$ Angle measured by boom inclinometer

$\theta_{2} \quad$ : Angle measured by stick inclinometer

$\theta_{3}:$ : Angle measured by bucket inclinometer

$\theta_{\text {bucket }}:$ Bucket joint angle

$\theta_{\text {stick }}$ : Stick boom joint angle

$e_{\text {tot }}$ : Total position error

\begin{abstract}
$e_{\text {slew }}:$ Relative error in slew position
$e_{\text {boom }}:$ Relative error in base boom position

$e_{\text {stick }}$ : Relative error in stick boom position

$e_{\text {bucket }}$ : Relative error in bucket position
\end{abstract}

\section{INTRODUCTION}

Hydraulic excavators are widely used in earth-moving tasks, for example digging and grading. The working conditions of the machine operator are often uncomfortable because of noise, vibration, and 
repetitive nature of the tasks. Because demand for excavator operators is sensitive to economic fluctuations and training takes a lot of time, it is sometimes difficult to find skilled excavator operators for every construction project.

Intelligent control systems could ease all these problems by performing some tasks for the operator or even enabling autonomous excavation. Moreover, as hydraulic excavators are typically very heavy-duty machines, the extra cost of an intelligent sensor and control system is very little in contrast to the total cost of the machine. The recent price development of raw materials and, on the other hand, electronic components makes the ratio even smaller.

A small wheel loader with an excavator attachment is presented as an example in this paper. A cost-effective control system with operator assisting functions is installed and tested. The developed control system enables automatic bucket emptying and return. Preliminary results are presented and future work is discussed.

The basic control concept appears in several patents $[5,6]$. However, the patented systems are rather expensive and complicated. There has also been significant development in sensor technology since the patents have been published. Therefore, this research presents a more practical approach with modern components.

\section{TEST EQUIPMENT}

The test equipment consists of a small commercial wheel loader and an excavator attachment. The machine suits research use well: It is compact and it has mechanical and hydraulic structures similar to larger machines. A photograph of the machine is shown in Figure 1.

\section{Small Wheel Loader}

The wheel loader is modified from Avant Tecno 300 series [1]. The machine is skid steered and has a hydrostatic power transmission. The engine is a Kubota diesel with a power output of $15 \mathrm{~kW}$. There is a variety of attachments available for the machine.

The original machine has mechanically operated valves which have been replaced by an electronically controlled stackable mobile proportional valve with six spools. In previous research a CANopen-based distributed control system has been developed. [7] Connecting the attachments is easy: There are quick couplings for hydraulic power and a multipole connector for electric supply and CAN.

\section{Excavator Attachment}

The excavator attachment is modified from Avant Backhoe 205. It has four hydraulic cylinders for slew, boom, stick, and bucket movements. The original mechanically operated valves have been replaced like the ones of the wheel loader. An electronic control unit for the excavator has been designed. The control unit can be configured to compensate typical nonlinear characteristics of proportional valves. [4]

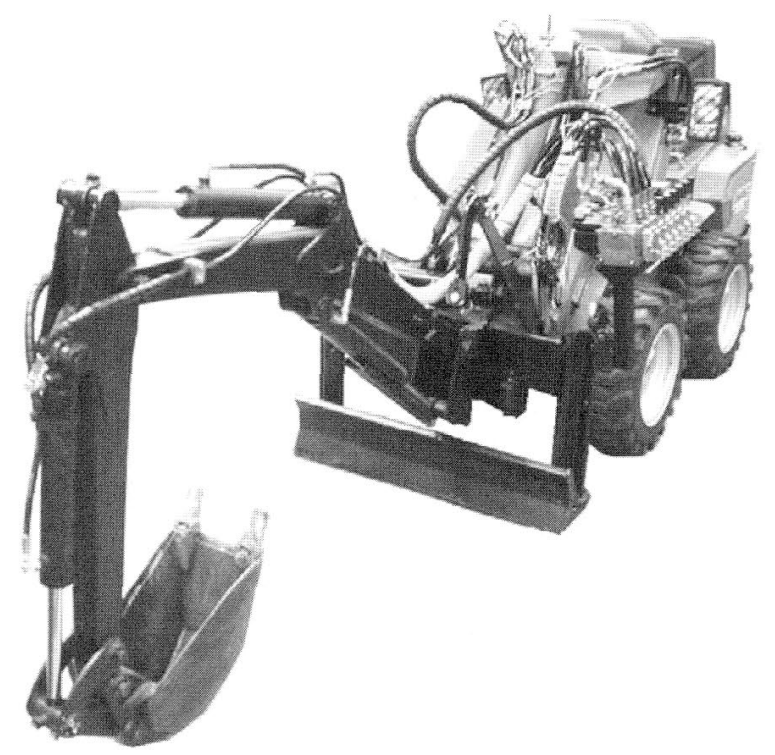

Figure 1 Wheel loader with excavator attachment

In previous research the control unit was programmed to perform simple closed-loop control of the slew cylinder with a linear cylinder position sensor [3]. Because the results were encouraging, more sensors have been added. In addition to the slew cylinder sensor, there are now three inclinometers measuring the absolute positions of the main boom, the stick boom, and the bucket. The arrangement is shown in Figure 2 where the boom, stick, and bucket inclinometers are marked with numbers 1,2, and 3 , respectively. The inclinometer module has been developed at the Department of Intelligent Hydraulics and Automation [2].

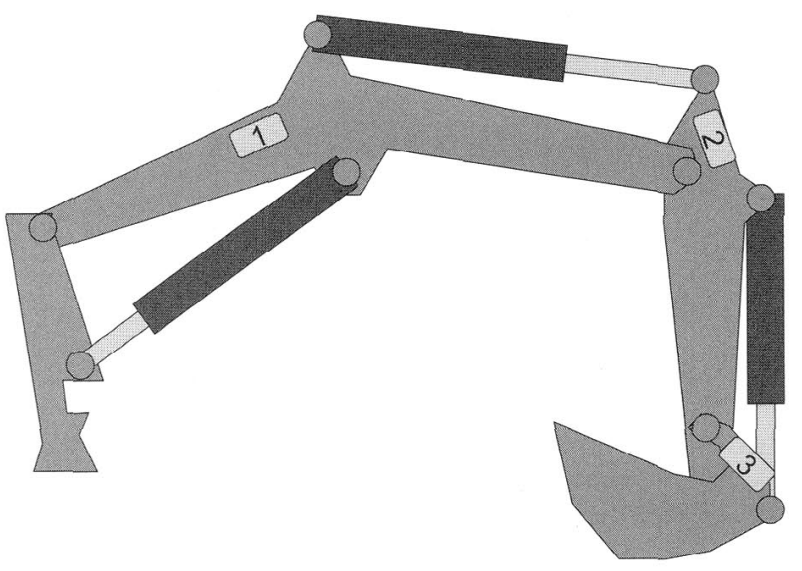

Figure 2 Inclinometer installations on excavator 
The inclinometers have MEMS accelerometers which have a limited bandwidth. This should not, however, be a problem with this application. The inclinometers also have MEMS gyroscopes that can be enabled in case the bandwidth has to be extended. The inclinometers have a CAN interface so they are simply connected using 4-pole cables that supply power and communications.

Because there is no inclinometer installed on either the wheel loader or the excavator frame, the actual joint angle of the base boom can not be calculated. This is, however, not a problem in this study as long as the excavator is not pressed against the ground so that the entire machine is lifted.

\section{CONTROLLER DESIGN}

As the first step in developing an autonomous control system a simple closed loop controller for slew movement was used. The operator was able to store desired trajectory points and command the slew cylinder to follow them. Now the concept is extended to all four cylinders, which enables automatic emptying and return of the bucket, for example.

A block diagram of the excavator controller is shown in Figure 3. The valve control signals are produced with PWM outputs of the excavator control unit. The valves can be controlled directly with open loop signals. The signals are processed according to parameters that can be set using the CANopen interface.

The controller also approximates the total volume flow that is required from the wheel loader based on the sum of spool control signals of the excavator.

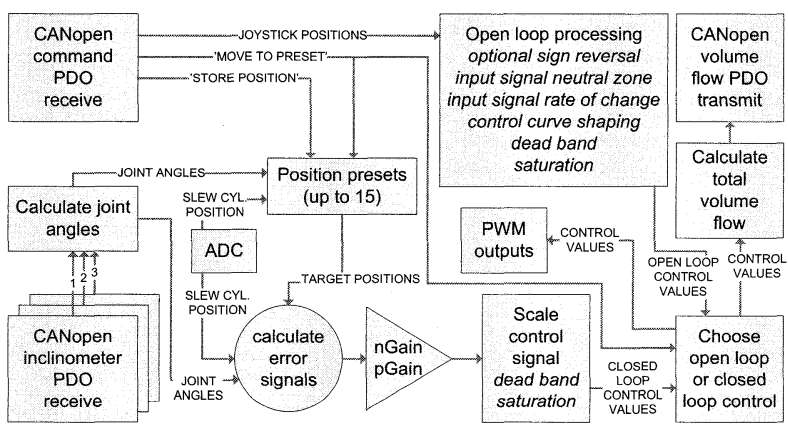

Figure 3 Excavator controller block diagram

Because the inclinometers measure the angle relative to gravity vector, the controller calculates the difference between two inclinometers over a joint to obtain the joint angle according to Eq. (1) and Eq. (2).

$$
\begin{aligned}
& \theta_{\text {bucket }}=\theta_{3}-\theta_{2} \\
& \theta_{\text {stick }}=\theta_{2}-\theta_{1}
\end{aligned}
$$

Since there are only three inclinometers, the angle of the base boom is obtained directly from the signal of the inclinometer 1. This causes some offset if the wheel loader is not carefully levelled. However, if the wheel loader keeps steady, the offset is not a problem at this stage of the research because the trajectory points have the same offset when they are stored.

Because the inclinometers are based on accelerometers, there is some disturbance in the angle signals as the machine vibrates or the bucket is hit against the ground. Therefore, the maximum angular velocity of the signals is limited to $200 \%$. The effect of the rate limiter on the bucket angle is shown in Figure 4.

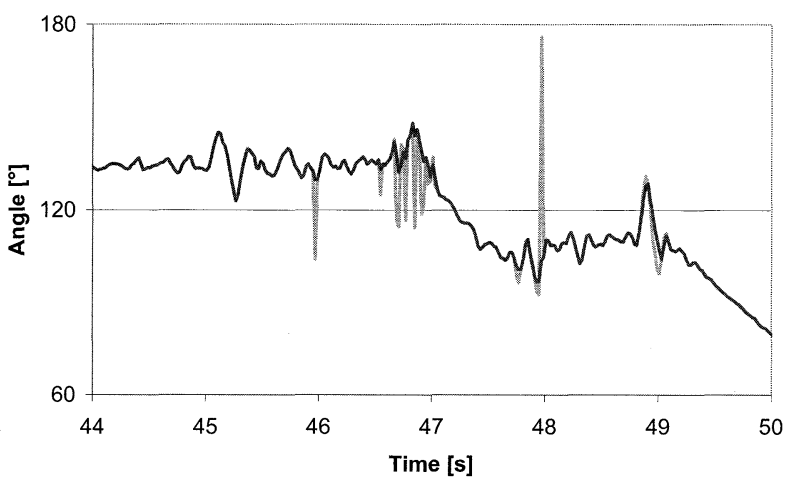

Figure 4 Effect of rate limiter on bucket angle signal

Up to 15 trajectory points can be stored during open loop control. When commanded, the controller will drive the excavator into a desired preset position. The position controller has an individual proportional controller for each cylinder. This typically results in a slightly curved bucket trajectory. There is, however, no path following controller. Therefore, the trajectory points have to be selected so that the order of the joint movements if insignificant. A total error signal is calculated according to Eq. 3 .

$$
e_{\text {tot }}=\sqrt{e_{\text {slew }}^{2}+e_{\text {boom }}^{2}+e_{\text {stick }}^{2}+e_{\text {bucket }}^{2}}
$$

If the total error stays below a set limit a set settling time, the controller will switch back to open loop control. The parameters were set to $3 \%$ total relative positioning error and $500 \mathrm{~ms}$ settling time. These limits were experimentally found suitable for the excavation application.

\section{TEST SETUP}

The control system of the wheel loader was configured to send a 'store position' command when a button on the user interface is pressed. Another button was configured to send a 'move to preset' command. The number of the 
preset was set to cycle from 1 to 3 for both commands, which enabled bucket emptying and return functionality:

1. over digging point, ready to fill bucket

2. soil in bucket, boom over emptying position

3. stick boom and bucket at emptying position A fourth trajectory point could be introduced if the soil is dug so deep that the base boom has to be lifted before sending the 'move to preset 2' command. Anyway, the three trajectory points are enough to prove that the concept works.

Because the hydraulic system of the wheel loader has a fixed pump, the control system of the wheel loader was also programmed to control the diesel engine according to the volume flow request from the excavator.

The gain parameters of the proportional controllers were tuned experimentally. The main problem is that the excavator frame is not very steadily supported. Therefore, the controller gains have to be quite small to prevent the wheel loader from moving on the ground.

\section{RESULTS}

The system was tested by first doing one excavation cycle manually and storing the required trajectory points. Figure 5 shows the joint angle signals during the manual excavation cycle. The vertical dash lines show the three trajectory points that are stored. Note that returning the excavator over the digging point is not shown.

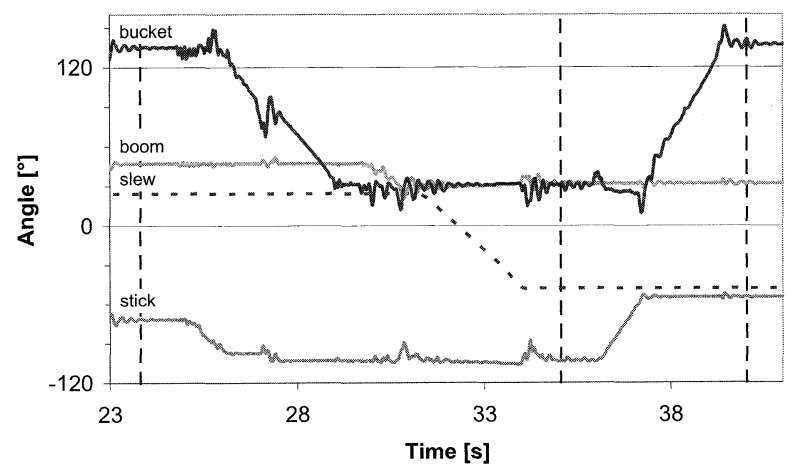

Figure 5 Manual excavation cycle

Between the stored points 1 and 2 the bucket is first filled using the bucket and stick cylinders and then moved over the emptying position using the boom and slew cylinders. Between the stored points 2 and 3 the bucket is emptied using the stick and bucket cylinders.

Figure 6 shows an excavation cycle that includes the automatic emptying and return of the bucket. Grey background indicates manual control. The vertical dash lines indicate the instant when a 'move to preset' command is sent and the fixed lines indicate when the preset is reached. The preset number 3 is actually not reached at all because the operator sends 'move to preset 1 ' as soon as the bucket has emptied at $80.5 \mathrm{~s}$.

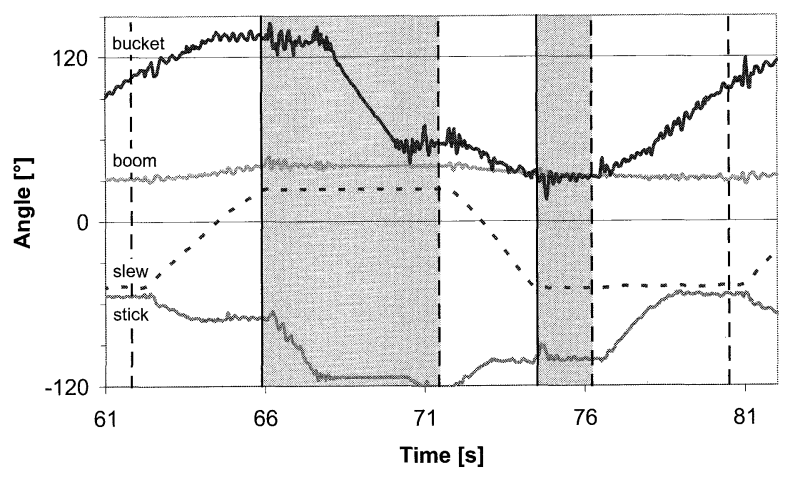

Figure 6 Excavation cycle with automatic emptying

\section{CONCLUSIONS}

Conventional mobile proportional valves and low-cost inclinometers can perform closed loop position control. The control software can be quite simple but there are several parameters to configure. The position controller can make excavation work easier and improve the efficiency of novice operators. The preliminary results of this paper could probably be further improved by choosing the controller gains, total error limit, and angular signal rate limits more carefully after thorough testing.

\section{REFERENCES}

1. Avant 300 series, 500 series, Avant Tecno Oy, Ylöjärvi, Finland.

2. Honkakorpi, J., CAN-bus based embedded inclinometer, Master's thesis, Tampere, Finland, 2007, 47 p. (in Finnish)

3. Karhu, O., Moya, J., Uusisalo, J. and Huhtala, K., Enabling autonomous functions on hydraulic excavator attachment, Proceedings of the SICFP, Tampere, Finland, 2007, pp. 35-45.

4. Karhu, O., Vilenius, J., Uusisalo, J. and Huhtala, K., Developing intelligent hydraulic excavator, Proceedings of the PTMC, Bath, UK, 2006, pp. 265-274.

5. Sutton, J. O., Computer controlled backhoe, US patent 4288196, 1981, $10 \mathrm{p}$.

6. Takayasu, I., Kazuo, O., Saburo, N., Michiaki, I., Toshiaki, H. and Kazuhiro, I., (Kabushiki Kaisha Komatsu Seisakusho), Semi-automatic hydraulic excavator, US patent 4377043, 1983, 6 p.

7. Vilenius, J., Raneda, A. and Huhtala, K., Characteristics of teleoperated skid steered mobile machine, Proceedings of the FPNI-PhD Symposium on Fluid Power, Terrassa, Spain, 2004, pp. 587-598. 